\title{
CALIDAD DE VIDA LABORAL EN PERSONAL DE ENFERMERIA: UNA REVISIÓN SISTEMÁTICA EN LA LITERATURA ACTUAL
}

\section{QUALITY OF WORKING LIFE IN THE NURSING STAFF: A REVIEW SYSTEMATIC IN THE CURRENT LITERATURA}

Recibido septiembre 2015 Aceptado enero 2016 Publicado noviembre 2016

Correspondencia: Villas del Padre, Zacatecas, Zacatecas, México, Teléfono 4921037789 lily.62@live.com.mx.

\section{Autores:}

Lilia Rodarte Cuevas

Alumna de la Maestría en Ciencias Biomédicas en la Universidad Autónoma de Zacatecas. Enfermera, Especialista en Enfermería Pediátrica y Administración y Docencia en Enfermería

Roxana Araujo Espino

Master en Enfermería. Doctora en Ciencias en Enfermería. Docente de la Universidad Autónoma de Zacatecas, México

Perla María Trejo Ortiz

Master en Enfermería. Doctora en Ciencias. Doctora en Ciencias en Enfermería. Docente de la Universidad Autónoma de Zacatecas, México

José González Tovar

Doctor en Ciencias. Docente de la Universidad Autónoma de Coahuila, México

Palabras clave: calidad de vida, trabajo, enfermería

Key words: quality of life, job, nursing 


\section{RESUMEN}

La presente investigación tuvo como objetivo analizar publicaciones nacionales e internacionales sobre el estado del arte de la calidad de vida laboral en personal de enfermería. Se realizó una revisión sistemática, en las bases de datos EBSCO, Scielo, PubMed y Redalyc, para identificar artículos publicados entre Enero del 2009 y Mayo de 2014. La muestra final quedó conformada por 21 artículos, la mayoría de los estudios fueron realizados en Brasil y Colombia, el mayor número de artículos se publicaron en el periodo de 2009-2017. Las publicaciones seleccionadas abordaron diferentes aspectos de la calidad de vida laboral como fueron: percepción o significado de la calidad de la vida profesional, condiciones laborales de las enfermeras, factores psicosociales asociados e impacto de la calidad de vida laboral en la percepción de la calidad de la atención.

Palabras clave: calidad de vida, trabajo, enfermería.

\section{ABSTRACT}

The present study aimed to analyze national and international publications on the State of the art of the quality of working life in nurses. He was a systematic review, EBSCO, PubMed and Scielo and Redalyc, databases to identify articles published between January 2009 and may 2014. The final sample consisted of 21 items, most of the studies were performed in Brazil and Colombia, the largest number of articles were published in the period 2009-2011. Selected publications addressed different aspects of the quality of working life as they were: perception or meaning of the quality of professional life, working conditions of nurses, associated psychosocial factors and impact of the quality of working life in the perception of the quality of care.

Keywords: quality of life, job, nursing.

\section{INTRODUCCIÓN}

lo largo y ancho del mundo para todo ser humano el trabajo y la familia son las principales
preocupaciones, aunque no fue siempre así. Antes, a los hombres les preocupaba el trabajo
y a las mujeres la familia, pero hoy, son muchas las mujeres que realizan un trabajo remunerado,
legando a integrar casi la mitad de la mano de obra a nivel mundial. Respecto al profesional de enfermería, estos representan el 70\% de la mano de obra de cualquier institución de salud, sea del orden público o privado, siendo en su mayoría mujeres y son los encargados de otorgar cuidados tendientes a la prevención, educación para la salud, curación y rehabilitación de la población en general, con un sentido ético, humanístico, con alta calidad y calidez en sus cuidados; sin embargo, y gracias al incremento de enfermedades crónico-degenerativas y la escasez de personal, las cargas de trabajo dentro de las instituciones de salud han aumentado. Lo anterior, aunado al ausentismo laboral, los bajos sueldos, la multiplicidad de empleos y la competitividad empresarial provocan altos índices de estrés, insatisfacción laboral, menor rendimiento y frustración personal, lo que deteriora la salud física y psíquica creando un importante desequilibrio en la combinación de trabajo y familia, lo que sin lugar a duda deteriora la calidad de vida profesional (1).

Para las instituciones de salud es de gran importancia reconocer los factores que inciden en la calidad de vida laboral ya que el trabajo es una actividad humana individual y colectiva que requiere de esfuerzos, aptitudes y tiempo donde los individuos llevan a cabo a cambio de compensaciones económicas y materiales, pero también psicológicas y sociales que contribuyen a sus necesidades (2). 
Por lo anteriormente descrito la presente revisión tiene como objetivo analizar publicaciones nacionales e internacionales sobre el estado del arte de la calidad de vida laboral en personal de enfermería.

\section{MATERIAL Y METODOS}

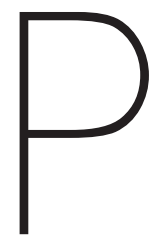

ara llevar a cabo la revisión sistemática de las publicaciones, se estableció un plan de trabajo de acuerdo con lo sugerido por la literatura (3): 1) El propósito de la investigación se instauró con la revisión de diferentes publicaciones, para lograr un conocimiento amplio sobre el tema; 2) Se determinaron los criterios de inclusión y exclusión de las distintas publicaciones para llevar a cabo la búsqueda; 3) Se delimito toda la pesquisa de utilidad de las publicaciones para elegir los artículos de interés para el tema; 4) Se examinaron los artículos; 5) Se llevó a cabo el razonamiento, discusión y conclusión de los descubrimientos, y 6) Se dieron a conocer los resultados.

Las publicaciones que se tomaron en cuenta fueron llevadas a cabo por expertos de distintas disciplinas del área de la salud y las ciencias sociales de diferentes países. Para realizar la búsqueda se procedió a investigar en las bases de datos, EBSCO, Scielo, PubMed y Redalyc, de manera adicional se acudió al buscador web Google Académico para encontrar artículos que no aparecieron en texto completo en las bases de datos. Se emplearon operadores boleanos dentro de los campos de título y resumen: Calidad de vida OR calidad de vida laboral OR profesional de enfermería OR percepción de la calidad de vida OR turnos nocturnos, al final de las pesquisas se identificaron 63 artículos y se comenzó la lectura de los títulos y resúmenes.

El criterio de inclusión para las indagaciones se delimitó a los artículos divulgados entre el mes de Enero de 2009 a Mayo de 2014, esto porque el estudio del tema de interés es muy amplio dentro del área de las ciencias sociales. Se eligieron artículos en inglés, español y portugués que estuvieran en texto completo y que incluyeran las variables de estudio. Se eliminaron artículos que se enfocaran al estudio de la satisfacción laboral y estrés en el trabajo, variables que comúnmente son confundidas con la calidad de vida laboral.

Debido a que el objetivo de la revisión fue conocer el estado del arte, se incluyeron artículos que correspondieran a los consiguientes niveles de investigación: I (estudios descriptivos, transversales, comparativos), II (no experimentales), III (exploratorio, diseño mixto quan-cuali), N (observacional e inferencial), V (cuantitativo-correlacional), VI (Cohorte, prospectivo), VII (epidemiológico, transversal).

Después de leer los títulos y resúmenes se tomaron en cuenta 32 artículos para llevar a cabo el análisis crítico de los mismos, buscando resultados que demostraran la relación entre la calidad de vida laboral y sus diferentes componentes y buscando la coherencia entre el propósito del estudio, el diseño, la metodología y los resultados. Para llevar a cabo el análisis se emplearon la lectura crítica y el subrayado. Posteriormente al análisis se eliminaron 11 artículos ya que no cumplieron con los criterios de interés para el estudio, quedando solamente 21 artículos que cumplieron con los requisitos establecidos presentando los resultados a continuación.

\section{RESULTADOS}

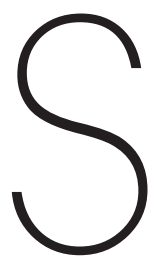
e encontró que en una mayor proporción los estudios se realizaron en países como Brasil (19.0\%) y Colombia (14.2\%). En relación a las fechas de publicación, once (52.8\%) artículos fueron publicados en el periodo 2009-2011, frente a diez (47.6\%) publicados en el periodo 2012-2014, con un nivel de evidencia N para estudios no experimentales y descriptivos-correlacionales y un factor de impacto de 0.03 a 1.8 . 
En cuanto a la población de estudio la mayor proporción de las investigaciones se realizaron en mujeres profesionales de enfermería con una edad promedio entre 30 y 40 años y un grupo menor de estudios fueron realizados en personal de salud en general.

Las publicaciones abordaron diferentes componentes de la calidad de vida laboral por lo que se agruparon en los siguientes apartados: percepción o significado de la calidad de la vida profesional, condiciones laborales de las enfermeras, factores psicosociales asociados e impacto de la calidad de vida laboral en la percepción de la calidad de la atención.

En cuanto a la percepción o significado de la calidad de vida profesional, las investigaciones difieren en sus resultados. Por un lado se encuentran aquellos estudios en los que se señala que el profesional de enfermería percibe como mala su calidad de vida, con porcentajes que varían de un 70\% a un $80 \%$, de la misma manera relacionan la mala calidad de vida laboral con el aspecto económico, la falta de recursos humanos y materiales y los horarios de trabajo(2)(4)(5). Por el otro, se encontraron estudios donde se evidencia que la mayoría de la población de interés percibe como buena su calidad de vida (89-91\%)(6). Es de resaltar que éste último grupo de investigaciones han sido realizados en países como México y Argentina.

Referente a las condiciones laborales del profesional de enfermería las publicaciones muestran que este aspecto de la calidad de vida laboral es deficiente con una media de 6, en situaciones como la retribución económica, la falta del recurso humano y material, infraestructura física, alta carga laboral y la calidad del trabajo físico, es importante tomar en cuenta que estos estudios se realizaron tanto en países de Asia como de Latinoamérica (7)(8)(9) (10)(11)(12)

Los factores psicosociales asociados a la calidad de vida profesional fueron estudiados nuevamente en países de Asia y Latinoamérica, específicamente Brasil, Colombia y China donde las publicaciones difieren entre sí, unos muestran que la calidad de vida laboral es mala en el ámbito social con un 40-50\% y con el estrato socioeconómico donde en el nivel 1 manifiestan nada de calidad de vida laboral en un 100\%, sin embargo otras reportan que la calidad de vida es bastante en un 55\% y la relación positiva de la misma con aspectos como el arraigo laboral y el afectivo(13)(14)(15)(16)(17)(18).

Por último el impacto de la calidad de vida laboral en la percepción de la calidad de la atención fue estudiado en países de América del Norte, donde las publicaciones reportan que las cargas de trabajo, la fatiga, el estrés, el turno laboral, el número de pacientes asignados, el entorno físico, el equilibrio entre el trabajo y el ocio, la seguridad en el empleo y el tiempo para su atención personal impactan su calidad de vida laboral, sin embargo se muestran altamente satisfechos con la atención que proporcionan al cliente(19)(20).

\section{CONCLUSIONES}

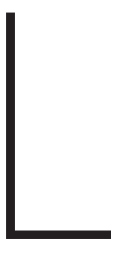

os hallazgos de la presente revisión muestran que la calidad de vida laboral del profesional de enfermería se percibe desde distintos puntos de vista, por una parte los estudios emargo otros reportan como mala la misma. esto puede deberse al tipo de institución donde laboran ya que existe evidencia de que por ejemplo en México el Instituto Mexicano del Seguro Social con sus políticas institucionales brinda condiciones laborales que permiten al trabajador se muestre satisfecho sobre todo en el aspecto económico, sin embargo existen instituciones de salud que no proporcionan las mismas condiciones, es por eso que las publicaciones analizadas muestran esa dualidad de la calidad de vida laboral del profesional de enfermería, también es importante destacar otros aspectos y que también han sido estudiados,

Enf Neurol Vol. 15. No. 1 enero - abril 2016 . N 
estos son los que se relacionan con las condiciones laborales y psicosociales del profesional de enfermería donde el ámbito social, el estrato socioeconómico, las condiciones del trabajo, los bajos salarios y la alta carga laboral impactan de manera importante en la calidad de vida laboral.

Esto puede deberse en gran medida a la infraestructura física y organizacional de las instituciones donde laboran, ya que el modelo neoliberal que ha alcanzado a distintos países sobre todo a los llamados subdesarrollados no permite mejorar las condiciones y prestaciones laborales que permitan el crecimiento personal, profesional y laboral que triaga como consecuencia esa calidad de vida laboral que impacte de manera importante en la calidad de la atención del usuario, es importante señalar que las publicaciones que estudiaron ese aspecto muestra que no importa que el profesional no cuente con las mejores condiciones para realizar su trabajo, aun así se muestran altamente satisfechos con la atención que proporcionan al cliente.

\section{CUADROS}

\section{1.- Características de los artículos seleccionados}

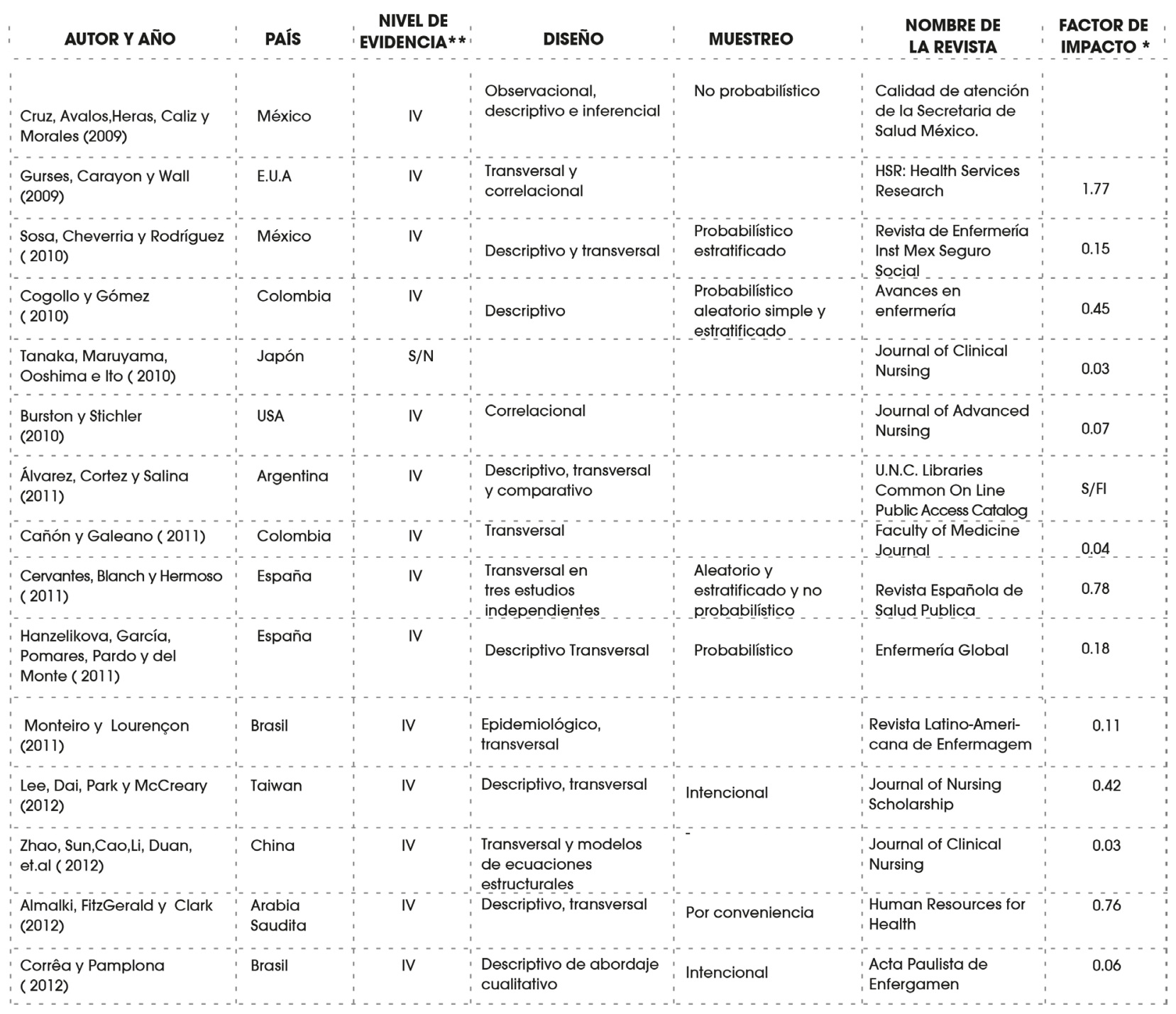




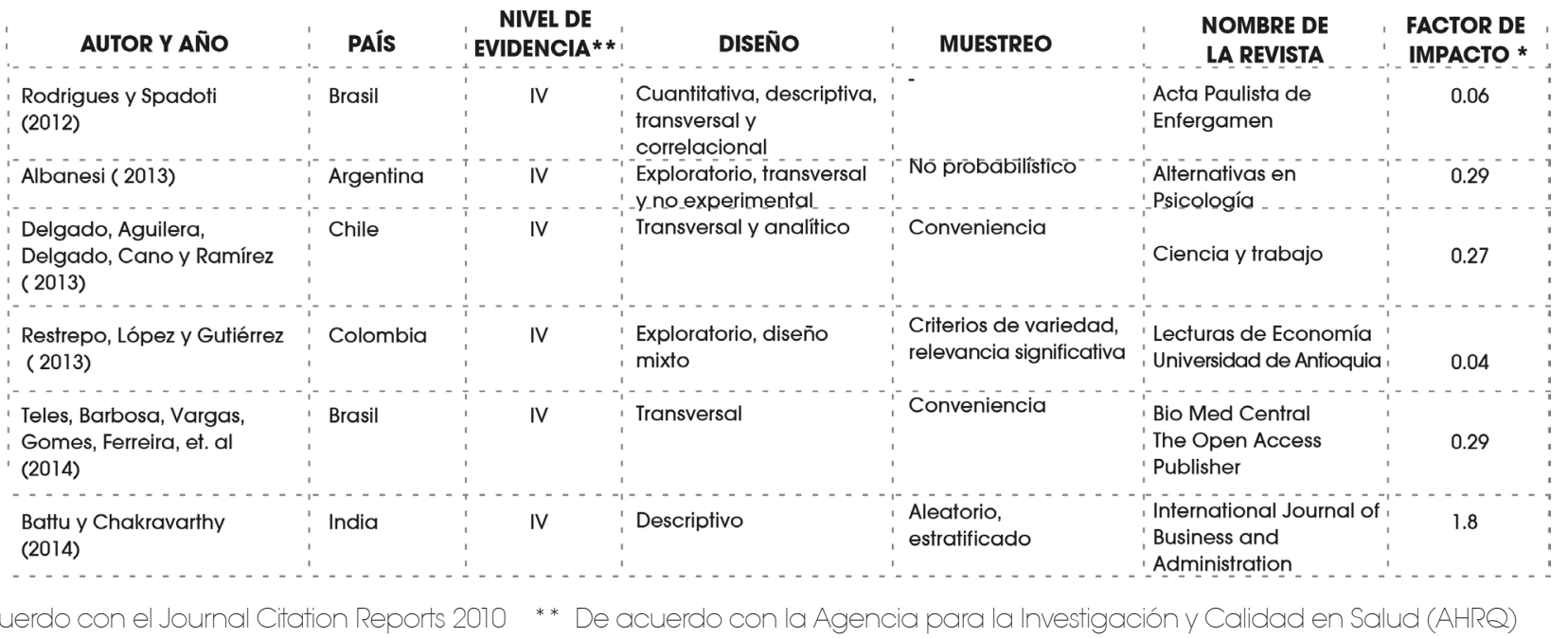

* De acuerdo con el Journal Citation Reports 2010 ** De acuerdo con la Agencia para la Investigación y Calidad en Salud (AHRQ)

\section{REFERENCIAS BIBLIOGRÁFICAS}

1. Duran MM. Bienestar psicológico: El estrés y la calidad de vida en el contexto laboral. Revista Nacional de Administración.2010 1(1): 71-84.Costa Rica.

2. Albanesi S., Percepción de calidad de vida profesional en trabajadores de la salud. Alternativas de Psicología. Revista Semestral. Tercera Época. Año XVII. Número 28. Febrero-Julio 2013. Argentina.

3. Mendes KD, Silveira RCCP, Galvão CM. Revisão integrativa: Método de pesquisa para a incorporação de evidências na saúde e na enfermagem. Texto Contexto Enferm. 2008; 17 (4): 758. Brasil.

4. Álvarez N., Cortez ME., Salina A., Calidad de vida profesional de la enfermeras. Tesis. U.N.C. Libraries, 2012, Argentina.

5. Cruz R., Avalos AG., Heras A., Caliz AP., Santos J. Calidad de vida profesional, su compromiso con la calidad de los trabajadores de la salud y el impacto en la acreditación. Avances No. 28 Volumen 9, 2009. México.

6. Sosa OR, Cheverria S., Rodríguez ME. Calidad de vida profesional del personal de enfermería. Rev Enferm Inst Mex Seguro Soc 2010; 18 (3): 153-158, México.

7. Delgado D., Aguilera MA., Delgado F., Cano I. y Ramírez O., Calidad de Vida en el Trabajo y Condiciones de Trabajo en Auxiliares de Esterilización. Cienc Trab, Sep-Dic; 15 (48): 148-151, 2013, Chile.

8. Almalki MJ., FitzGerald G., Clark M.,Quality of work life among primary health carenurses in the Jazan region, Saudi Arabia: a cross-sectional study. Almalki et al. Human Resources for Health 2012, 10:30. Arabia Saudita.

9. Battu N., Chakravarthy GK., Quality of work life of nurses and paramedical staff in hospitals. International Journal of Business and Administration Research Review, Vol.2, Issue.4, Jan-March, 2014. India.

10. Lourençon CF., Monteiro Ml., Condiciones de vida y trabajo de los profesionales en un Servicio de Atención Móvil de Urgencia. Rev. Latino-Am. Enfermagem 19(4); 07 jul-ago, 2011 . Brasil.

11. Restrepo F., López AM., Gutiérrez LM., La calidad de vida laboral del personal de salud del Uraba antioqueño. Lecturas de Economía Universidad de Antioquia. Colombia.

12. Lee YW., Dai YT., Park CHG., McCreary LL., Predicting Quality of Work Life on Nurses "Intention to Leave. Journal of Nursing Scholarship, 2013; 45:2, 160fi168.Taiwan.
13. Rodrigues D., Spadoti RA., Quality of Work Life and Work-Related Musculoskeletal Disorders among Nursing Professionals. Acta Paul Enferm. 2012; 25(5):701-7. Brasil.

14. -Cañón SC., Galeano G., Factores laborales psicosociales y calidad de vida laboral de los trabajadores de la salud de ASSBASALUD E. S.E. Archivos de Medicina Volumen 11 N²- Julio-Diciembre de 2011 . Colombia.

15. Cogollo Z. y Gómez E., Condiciones Laborales en Enfermeras. av.enferm., XXVIII (1): 31-38, 2010, Colombia.

16. Corrêa D., Pamplona VL., Quality of work life of nurses in primary health care. Acta Paul Enferm. 2012; 25(2): 277-83. Brasil.

17. Zhao XG., Sun T., , Cao QR., Li C., Duan XJ., et al. The impact of quality of work life on job embeddedness and affective commitment and their co-effect on turnover intention of nurses. Journal of Clinical Nursing, 22, 780fi788, 2012. China

18. Teles MA., Barbosa MR., Vargas AM., Gomes VE., Ferreira E., et al. Psychosocial work conditions and quality of life among primary health care employees: a cross sectional study. Health and Quality of Life Outcomes 2014, 12:72. Brasil.

19. Burtson PL., \& Stichler JF., Nursing work environment and nurse caring: relationship among motivational factor. Journal of Advanced Nursing 66(8), 1819fi1831.USA.

20. Gurses AP. ,Carayon P.,Wall M., Impact of Performance Obstacles on Intensive Care Nurses, Workload, Perceived Quality and Safety of Care and Quality of Working Life. HSR: Health Services Research 44:2, Part I (April 2009).USA.

21. Tanaka S., Maruyama Y., Ooshima S., Ito H., Working condition of nurses in Japan: awareness of workfilife balance among nursing personnel at a university hospital. Journal of Clinical Nursing, 20, 12fi22, 2010. Japón.

22. Cervantes G. , Blanch JM. , Hermoso D., Calidad de vida laboral en centros asistenciales de salud catalanes. Arch Prev Riesgos Labor 2011; 14 (1): 13-19. España.

23. Hanzelikova A., García MV., Pomares M., Pardo MJ., Del Monte J., La calidad de vida profesional de las enfermeras en geriatría. Enfermería Global Revista Electrónica Trimestral de Enfermería N 24 Octubre 2011. España.

Enf Neurol Vol. 15. No. 1 enero - abril 2016 